\title{
The Inhibiting Effect of Abscisic Acid on Fragrance of Kam Sweet Rice
}

\author{
Zheng Kong ${ }^{1,2}$, Degang Zhao ${ }^{1,2,3, *}$ \\ ${ }^{1}$ Guizhou Key Laboratory of Agro-Bioengineering, Guizhou University, South Campus, Huaxi, Guiyang City, Guizhou Province, \\ People's Republic of China \\ ${ }^{2}$ Key Laboratory of Green Pesticide and Ago-Bioengineering, Ministry of Education, Guizhou University, North Campus, Huaxi, \\ Guiyang City, Guizhou Province, People's Republic of China \\ ${ }^{3}$ College of Life Science, Guizhou University, South Campus, Huaxi, Guiyang City, Guizhou Province, People's Republic of China \\ *Corresponding author: dgzhao@gzu.edu.cn
}

Received April 08, 2014; Revised April 22, 2014; Accepted May 03, 2014

\begin{abstract}
The Asian cultivated rice (Oryza sativa L.) is a major type of cultivated rice in Asia [1]. The Kam sweet rice reported on Science have been able to preserve their varieties against genetic contamination from Indica strains [2]. Gou Cengao is an indigenous aromatic Kam sweet rice variety of China. This study aimed to characterize the effect of abscisic acid(ABA) on volatile compounds in grains of Gou Cengao. Using GC-O and SPME-GC-MS, nonanal displayed the highest odor activity value (OAV) in the grains and decreased after applicaton of $A B A$ in the rice seedlings. The relative expression of rice OsLOX3 (rice lipoxygenase 3) and OsHPL1 (rice hydroperoxide lyase 1) was assessed by RT-PCR in grains of Gou Cengao and the non-aromatic Lailong rice after pollination. Our data showed that OsLOX3 was elevated in Gou Cengao compared with the non-aromatic rice. In agreement, lipoxygenase (OsLOX3 gene product) levels and activity were elevated in aromatic rice samples. Interestingly, a significant negative Pearson correlation (-0.945) was found between the concentrations of endogenous ABA and the relative expression of rice OsLOX3 $(p<0.01)$. The results suggest that the inhibiting effect of ABA on the biosynthetic pathway of nonanal.
\end{abstract}

Keywords: Kam sweet rice, nonanal, 2-Acetyl-1-pyrroline, lipoxygenase activity, abscisic acid, OsLOX3

Cite This Article: Zheng Kong, and Degang Zhao, "The Inhibiting Effect of Abscisic Acid on Fragrance of Kam Sweet Rice.” Journal of Food and Nutrition Research, vol. 2, no. 4 (2014): 148-154. doi: 10.12691/jfnr-2-4-3.

\section{Introduction}

The Asian cultivated rice (Oryza sativa L.) is one of the most important crops and a major food source for more than half of the global human population [1]. Among all rice varieties produced worldwide, aromatic Kam sweet rice is known for its pleasant fragrance and taste. Kam sweet rice [2] is a major type of cultivated rice with a long history of cultural and culinary importance in Asia [3]. Indigenous aromatic Kam sweet rice types cultivated in China include Gou Cengao, Gou Gaoqian and Congjiang, found especially in southwest China where Kam sweet rice cakes are traditional foods. Gou Cengao has been cultivated for hundreds of years. However, major aromatic components found in the grains of this important rice have not been characterized.

Multiple studies have been carried out to describe factors that influence the production of volatile components. For instance, trace amounts of gibberellic acid result in decreased 2-acetyl-1-pyrroline levels in aromatic rice [4], and it has been reported that many aromatic components are markedly noticed in aromatic rice after treatment with abscisic acid (ABA) [5]. In addition, environmental conditions affect the aroma quality of aromatic rice: rice cropped in rain-fed paddy fields shows higher content in aromatic compounds [6]; the yield of aromatic volatile compounds is affected by salinity $[7,8,9]$. Furthermore, enzyme content is an important factor in aromatic compound synthesis. Indeed, 2-acetyl-1-pyrroline levels were regulated by the expression of $\Delta^{1}$-pyrroline-5-carboxylixc acid synthetase OsP5CS [10] and it has been shown that volatile aldehydes are produced through lipid oxidation and peroxidation [11]. Finally, Tomio et al. reported that 2acetyl-1-pyrroline content in rice was higher when ripened at low temperature (day: $25^{\circ} \mathrm{C} /$ night: $20^{\circ} \mathrm{C}$ ) compared with high temperature (day: $35^{\circ} \mathrm{C} /$ night $30^{\circ} \mathrm{C}$ ) (Itani et al. 2004). Likewise, reduction of temperature was suggested as an alternative way to retard aroma changes [12].

In this study, concentration of ABA and the volatile components of cooked Gou Cengao and rice grains of filling stage were analyzed to reveal the correlation between ABA and the key volatile compound.

\section{Materials and Methods}

Plant Materials. The aromatic (Gou Cengao) and nonaromatic (Lailong) Kam sweet rice types are Chinese aromatic japonica rice found in the Guizhou Plateau, 
which is located in the provinces of Yunnan and Guizhou in southwest China. All rice grains were milled to remove bran layers, and samples were sealed in zip lock bags and kept at $-80^{\circ} \mathrm{C}$ until use. The fifteen-day-old seedling with three leaves were either treated with water supplemented with $110 \mathrm{mg} / \mathrm{L} \mathrm{ABA}$ or with water alone for 48h. The green leaves were harvested and samples of equal weight (1.0 g) were homogenized in liquid nitrogen for the assay of volatile components.

Rice cooking. Aromatic Kam sweet rice Gou Cengao was cooked according to previous reports [13]. Briefly, $100 \mathrm{~g}$ sample of matured grains were added to $100 \mathrm{~mL}$ distilled water in a $1 \mathrm{~L}$ glass beaker and heated using an electromagnetic heater for $20 \mathrm{~min}$ at $100^{\circ} \mathrm{C}$. During heating the glass beaker was sealed by a special ground glass lid equipped with an exhaust port covered with aluminum foil. After cooking, the beaker and content were placed in $80^{\circ} \mathrm{C}$ water bath for the SPME step.

SPME Sampling. An equilibration time of 10-15 min in headspace solid-phase microextraction has been shown sufficient for most volatile compounds. Hence, SPME was carried out for 15 min using a 50/30 $\mu \mathrm{m}$ DVB SPME fiber (2 cm) attached to a manual holder from Supelco (Bellefonte, PA, USA). Then, the SPME fiber was desorbed for $5 \mathrm{~min}$ on an Agilent 5975C/6890 GC equipped with a HP 5973 mass-selective detector (Agilent Technologies, Palo Alto, CA, USA) and a $30 \mathrm{~m} \times 0.25$ $\mathrm{mm} \times 0.25 \mu \mathrm{m}$ Zebron ZB-5MSI fused silica capillary column (5\% Phenyl-95\% Dimethylpolysiloxane) from Zebron (USA). The injector temperature was kept at $250^{\circ} \mathrm{C}$ throughout the separation.

Solvent Extraction of Volatile compounds. Solvent extraction was used for samples during rice filling stages. $1 \mathrm{~g}$ fresh rice grains was ground and resuspended in $2 \mathrm{~mL}$ of a 1:1 dichloromethane and ethanol mixture, in a sealed screw cap vial $(22.5 \times 46 \mathrm{~mm})$ equipped with PTFE silicon septa (WAJR, China). The mixture was heated in a water bath at $60^{\circ} \mathrm{C}$ for 24 hours and the sample allowed to cool down at room temperature for $30 \mathrm{~min}$. Triplicate samples were analyzed GC-MS as described below.

\subsection{Gas Chromatography-Mass Spectrometry (GC-MS) and Gas Chromatography- Olfactometry (GC-O)}

GC-MS experiments were carried out on an Agilent 6890/HP 5973 instrument described above. Samples were injected in split mode. The column temperature was programmed to start at $40^{\circ} \mathrm{C}$ with a 2 min hold followed by increase at incremental rates of $4^{\circ} \mathrm{C} / \mathrm{min}$ to $150^{\circ} \mathrm{C}$. The interface temperature was $280^{\circ} \mathrm{C}$ and the flow rate of the carrier gas-helium was $1.0 \mathrm{~mL} / \mathrm{min}$. Volatiles were split between the mass spectrometer and the olfactory detection port (Gersterl ODP2, Germany) for description and intensity assessment. The mass spectrometer was operated in an electron impact (EI) ionization mode with electron energy of $70 \mathrm{eV}$ and electron multiplier voltage of $1052 \mathrm{~V}$. The temperature of the ion source was $230^{\circ} \mathrm{C}$ and mass spectra were obtained by scanning from m/z 20-450. For olfactory detection, three independent assessors trained for odor description of volatiles evaluated the samples. Odor intensity was classified as nd (not detected), 1 (very weak), 2 (weak), 3 (intermediate), 4 (strong), and 5 (very strong).
The volatiles perceived by all assessors were considered odor active components.

\subsection{Extraction of ABA}

The 1g sample was freeze-dried and separately ground in $11 \mathrm{~mL}, 5 \mathrm{~mL}, 2 \mathrm{~mL}, 2 \mathrm{~mL}$ of ethanol mixture (80\%) in the weak light. The homogenate were stored at $60^{\circ} \mathrm{C}$ for $12 \mathrm{~h}$ and submitted to centrifugation $\left(4^{\circ} \mathrm{C}, 5000 \mathrm{rpm}, 10\right.$ min). Using vacuum freezing, centrifugation and enrichment, the ethanol was removed from the supernatant fluid. In the next step the supernatants were added with 8 $\mathrm{mL}$ solution of ammonium acetate $(0.1 \mathrm{~mol} / \mathrm{L}, \mathrm{pH} 9.0)$ and the samples submitted to centrifugation $\left(4^{\circ} \mathrm{C}, 15000 \mathrm{rpm}\right.$, 20min). After filtering with polyvinylpyrrolidone (PVPP) column and diethyl amino ethyl sephadex gel (DEAE Sephadex A-25) column, the resulting samples collected by Sep-Pak C18 column $(1 \mathrm{~cm} \times 15 \mathrm{~cm}, 30 \mathrm{~mL})$ were eluted by $50 \%$ methanol solution for the next HPLC analysis.

\subsection{Identification and Quantification of Volatile Compounds and ABA}

The following standards were used for identification of aromatic compounds in cooked rice samples and ABA in rice seedlings: hexanal, furfural, heptanal, $(E)$-2-heptenal, 1-octen-3-ol, 2-pentylfuran, octanal, (E)-2-octenal, 1octanol, nonanal, decanal, $(E, E)-2,4$-nonadienal, and geranylacetone were purchased from Aladdin-reagent (China); benzaldehyde and 3-octen-2-one were provided by Xiyashiji (China) and 2-acetyl-1-pyrroline by J\&K Chemical (China). The n-alkanes $\mathrm{C}_{6}-\mathrm{C}_{19}$ (AccuStandard, USA) were used to derive the retention indices. ABA was provided by Aladdin-reagent (China).

All aromatic compounds of rice were identified by matching with mass spectra in the NIST and WILEY libraries and confirmed by comparing retention times with that of authentic standards. For quantification of each compound, standard curves were determined by analyzing GC-MS data after injection of different concentrations (ppm) or the compound diluted in hexane (Table 1).

For samples obtained by solvent extraction, nonanal was identified and quantified as described above, using the NIST and WILEY libraries. The following equation was obtained for the standard curve: $y=9 \times 10^{-6} \mathrm{x}+24.078$, with $\mathrm{r}^{2}=0.9999$, a validation comprised between 25 and $155 \mu \mathrm{g} / \mathrm{kg}$ and an RSD of $2.69 \%$.

For HPLC assay, the mobile phases used were the mixed solution of acetonitrile and $0.5 \%$ triethylamine (pH3.5). The first eluant and second eluant were respectively in $100 \%$ triethylamine and mixed solution (82\% triethylamine and $18 \%$ acetonitrile) with interval time $6 \mathrm{~min}$. The third eluant was $64 \%$ triethylamine and $36 \%$ acetonitrile after $18 \mathrm{~min}$. The program was set to flow velocity $1 \mathrm{~mL} / \mathrm{min}$, determine wavelength $210 \mathrm{~nm}$ and column temperature $35^{\circ} \mathrm{C}$. The injection volume of sample was $10 \mu \mathrm{L}$ with $80 \mu \mathrm{L}$ solution of $20 \%$ acetonitrile and $80 \%$ triethylamine $(0.4 \%, \mathrm{pH} .5)$. The gradient condition of ABA included $10 \mathrm{ng} / \mathrm{g}, 30 \mathrm{ng} / \mathrm{g}, 60 \mathrm{ng} / \mathrm{g}, 120$ ng/g, $240 \mathrm{ng} / \mathrm{g}, 350 \mathrm{ng} / \mathrm{g}, 500 \mathrm{ng} / \mathrm{g}$. The equation was obtained for the standard curve of ABA: $y=$ $0.15928 x+0.33493$, with $r^{2}=0.9999$, a validation comprised between 30.3 and $350 \mathrm{ng} / \mathrm{g}$ and RSD of 1.88\%. 
Table 1. Linearity, Sensitivity and Precision of Major Odor-Active Compounds detected in Cooked Aromatic Kam sweet rice Sample in grains during filling stage

\begin{tabular}{|c|c|c|c|c|}
\hline Compound & Standard Curve & $\mathrm{R}^{2}$ & Validation Range & $\mathrm{RSD}(\%)$ \\
\hline hexanal & $y=9 \times 10^{-6} x+2.5011$ & 0.9997 & 2.4-198 (ng/L) & 1.55 \\
\hline furfural & $y=7 \times 10^{-6} x+2.0448$ & 0.9993 & 3.7-175 (ng/L) & 1.73 \\
\hline heptanal & $y=5 \times 10^{-6} x+0.9388$ & 0.9993 & 2.7-196 (ng/L) & 3.74 \\
\hline 2-acetyl-1-pyrroline & $y=9 \times 10^{-7} x+0.0402$ & 0.9997 & 0.04-4.9 (ng/L) & 3.48 \\
\hline (E)-2-heptenal & $y=4 \times 10^{-6} x+3.2122$ & 0.9997 & $2.8-2.02$ (ng/L) & 2.36 \\
\hline benzaldehyde & $y=2 \times 10^{-5} x-1.2988$ & 0.9996 & 2.6-190 (ng/L) & 0.98 \\
\hline 1-octen-3-ol & $y=9 \times 10^{-6} x+0.5793$ & 0.9999 & $2.9-180$ (ng/L) & 3.19 \\
\hline 2-pentylfuran & $y=6 \times 10^{-6} x-0.0862$ & 0.9997 & 2.8-190 (ng/L) & 2.22 \\
\hline octanal & $y=6 \times 10^{-6} x-2.7271$ & 0.9994 & $2.1-150$ (ng/L) & 1.93 \\
\hline 3-octen-2-one & $y=3 \times 10^{-6} x+1.9795$ & 0.9999 & 2.4-204 (ng/L) & 1.58 \\
\hline (E)-2-octenal & $y=5 \times 10^{-6} x+3.7986$ & 0.9997 & 2.9-218 (ng/L) & 2.31 \\
\hline 1-octanol & $y=5 \times 10^{-6} x+4.3261$ & 0.9996 & 3.3-195 (ng/L) & 2.21 \\
\hline nonanal & $y=1 \times 10^{-5} x+18.383$ & 0.9992 & 24-199 (ng/L) & 0.29 \\
\hline decanal & $y=7 \times 10^{-6} x-0.5105$ & 0.9999 & $1.0-200(\mathrm{ng} / \mathrm{L})$ & 2.18 \\
\hline$(E, E)-2,4$-nonadienal & $y=3 \times 10^{-5} x-0.7644$ & 0.9991 & 2.8-190 (ng/L) & 1.48 \\
\hline geranylacetone & $y=2 \times 10^{-6} x+2.9497$ & 0.9997 & 2.6-195 (ng/L) & 0.87 \\
\hline
\end{tabular}

RNA isolation and semi-quantitative RT-PCR. Total RNA was isolated from rice grain samples obtained from aromatic Kam sweet rice Gou Cengao and non-aromatic rice Lailong. RNA extraction from ten independent samples was carried out at four distinct stages of the rice grain development $(6,8,10$, and 15 days after rice flowering), using the E.Z.N.A ${ }^{\mathrm{TM}}$ Plant RNA Kit (OMEGA, USA) and following manufacturer's instructions.

1. Collect frozen ground plant tissue(up to $100 \mathrm{mg}$ ) in a microfuge tube and immediately add $500 \mu \mathrm{L}$ Buffer RCL/2-mercaptoethanol. We recommend starting with 50 $\mathrm{mg}$ tissues at first. If results obtained are satisfactory increase amount of starting material. Samples should not be allowed to thaw before Buffer RCL/2-mercaptoethanol is added. Vortex vigorously to make sure that all clumps are dispersed. RNA cannot be effectively extracted from clumped tissue.

2. Incubate at $55^{\circ} \mathrm{C}$ for1-3 minutes. Centrifuge at maxi speed $(14000 \times \mathrm{g})$ for $5 \mathrm{~min}$ at room temperature.

3. Transfer the supernatant directly into a gDNA Filter Column in $2 \mathrm{~mL}$ collection tube and centrifuge at $14000 \times$ $\mathrm{g}$ for $2 \mathrm{~min}$ at room temperature.

4. Add eqaul volume Buffer RCB to the flow-through and mix well by pipetting up and down 5-10 times.

5. Apply one half of the mixture from step 4 to a HiBind ${ }^{@}$ RNA Mini column assembled in a clean $2 \mathrm{~mL}$ collection tube (supplied). Centrifuge at $10000 \times \mathrm{g}$ for 1 min at room temperature. Discard the flow-through liquid and place the column back into the collection tube.

6. Apply the regaining of the mixture from step 4 to the column. Centrifuge at $10000 \times \mathrm{g}$ for $1 \mathrm{~min}$ at room temperature. Discard the flow-through liquid and place the column back into the collection tube.

7. Add $400 \mu \mathrm{L}$ RWC Wash Buffer and centrifuge as above. Discard both flow-through liquid and collection tube.

8. Place column in a clean $2 \mathrm{~mL}$ collection tube (supplied), and add $500 \mu \mathrm{L}$ RNA Wash Buffer II diluted with ethanol. Centrifuge as above and discard flowthrough. Re-use the collection tube in step 9.

9. Wash column with a second $500 \mu \mathrm{L}$ RNA Wash Buffer II by repeating step 8. Centrifuge as above and discard flow-through. Then with the collection tube empty, centrifuge the column for $2 \min$ at $10000 \times \mathrm{g}$ to completely dry the column matrix.
10. Elution of RNA: Transfer the column to a clean 1.5 $\mathrm{mL}$ microfuge tube (not suplied) and elute the RNA with 30-50 $\mu \mathrm{L}$ of DEPC water (suplied). Make sure to add water directly onto column matrix. Incubate at room temperature for $2 \mathrm{~min}$. Centrifuge for $1 \mathrm{~min}$ at $10000 \times \mathrm{g}$. A second elution into the same tube may be necessary if the expected yield of RNA > $30 \mu \mathrm{g}$.

Reverse transcription was carried out separately for 10 $\min \left(25^{\circ} \mathrm{C}\right), 120 \mathrm{~min}\left(37^{\circ} \mathrm{C}\right)$ and $5 \min \left(85^{\circ} \mathrm{C}\right)$, using the high-capacity cDNA reverse transcription kit (Applied Biosystems, USA). To prepare the $2 \times$ RT master mix on ice (per $20 \mu \mathrm{L}$ reaction): $10 \times$ RT Buffer $2.0 \mu \mathrm{L}$; $25 \times$ dNTP Mix (100 mM) $0.8 \mu \mathrm{L} ; 10 \times$ RT Random Primers $2.0 \mu \mathrm{L}$; Multiscribe ${ }^{\mathrm{TM}}$ Reverst Transcriptase $1.0 \mu \mathrm{L}$; Rnase Inhibitor $1.0 \mu \mathrm{L}$; Nuclease-free $\mathrm{H}_{2} \mathrm{O} 3.2 \mu \mathrm{L}$. To prepare the cDNA Reverse Transcription reactions: 1 . Pipette $10 \mu \mathrm{L}$ of $2 \times$ RT master mix into each well of a 96well reaction plate or individual tube. 2. Pipette $10 \mu \mathrm{L}$ of RNA sample into each well, pipetting up and down two times to mix. 3. Seal the plates or tubes. 4. Briefly centrifuge the plate or tubes to spin down the contents and to eliminate any air bubbles. 5. Place the plate or tubes on ice until you are ready to load the thermal cycler.

Real-Time PCR was carried out on an ABI 7500 Fast Real Time PCR instrument with the 7500 v2.0.1 software (Applied Biosystems, USA) and Power SYBR Green PCR Master Mix (Applied Biosystems, USA). The manufacturer's instruction of Power SYBR Green PCR Master Mix: prepare the PCR reagents mix with Power SYBR Green PCR Master Mix $10 \mu \mathrm{L}$, forward primer $50 \mathrm{nM}$, reverse primer 50nM, template $100 \mathrm{ng}$, nuclease-free water. The total reaction volumn was $20 \mu \mathrm{L}$. Real-Time PCR conditions included an initial denaturation at $95^{\circ} \mathrm{C}$ for $10 \mathrm{~min}$ followed by 40 cycles of $95^{\circ} \mathrm{C}$ for $15 \mathrm{~s}$ and $60^{\circ} \mathrm{C}$ for $1 \mathrm{~min}$. The melting curves were obtained at $95^{\circ} \mathrm{C}$ for $15 \mathrm{~s}, 60^{\circ} \mathrm{C}$ for $1 \mathrm{~min}$; and $95^{\circ} \mathrm{C}$ for $15 \mathrm{~s}$. The following primers were used: OsLOX3 Forward, 5'-CAACAGGCTCTACATTCT-3'; OsLOX3 Reverse, 5'-GTGGCATAGGTGAAGATA-3' (Reference sequence: GenBank FJ660622.1); OsHPL1 Forward, 5'-AGCTCCTCCACAACCTCG-3'; OsHPL1 Reverse, 5'-CGATGTGTGGCAGGAAGAT-3' (Reference sequence: GenBank AK105964.1). The ubiquitin-conjugating enzyme E2 gene was employed as internal control: Forward primer 5'-CCGTTTGTAGAGCCATAATTGCA-3'; Reverse primer 5'-AGGTTGCCTGAGTCACAGTTAAGTG-3' 
(Reference sequence: GenBank AK059694.1). The mRNA levels were expressed relatively to the ubiquitinconjugating enzyme E2 gene in each sample.

Lipoxygenase Extraction and Enzyme Activity. Lipoxygenase was extracted as previously described [10]. Rice grains were frozen and ground in liquid nitrogen. The resulting powder was stored at $-20^{\circ} \mathrm{C}$ until use. For extraction, $1 \mathrm{~g}$ powder was added to $10 \mathrm{~mL}$ phosphate buffer (1\%PVP, pH6.5, 0.2M) and the suspension centrifuged at $10000 \times \mathrm{g}$ for $20 \mathrm{~min}\left(4^{\circ} \mathrm{C}\right)$. Ammonium sulfate was added to supernatants to achieve $35 \%$ saturation and the mixture centrifuged as described above. The resulting supernatants were precipitated with $70 \%$ $\left(\mathrm{NH}_{4}\right)_{2} \mathrm{SO}_{4}$ for $1 \mathrm{~h}$ and the samples submitted to centrifugation $\left(4^{\circ} \mathrm{C}, 10000 \times \mathrm{g}, 20 \mathrm{~min}\right)$. The pellet containing the enzyme was dissolved in $2 \mathrm{~mL}$ of $0.01 \mathrm{M}$ phosphate buffer (pH6.5) containing 10\% glycerol. The samples were desalinated by Sephadex G-25 column $(1 \mathrm{~cm} \times 15 \mathrm{~cm}, 30 \mathrm{~mL})$ and stored at $4^{\circ} \mathrm{C}$. Enzyme activity was determined as previously described [14]. Briefly, quartz color dishes were filled with $0.2 \mathrm{~mL}$ substrate, 2.75 $\mathrm{mL}$ phosphate buffer (pH6.5, 0.2M) and $50 \mu \mathrm{L}$ lipoxygenase extract and activity were assessed spectrophotometrically by monitoring the formation of conjugated dienes at $234 \mathrm{~nm}$. The enzyme activity unit was calculated as $\Delta \mathrm{A}_{234} \mathrm{~min}^{-1} \mathrm{~g}^{-1} \mathrm{FW}$. The substrate solution was prepared as proposed by Engeseth et al. [15]. Briefly, $0.1 \mathrm{~mL}$ linoleic acid stock solution was mixed with $2 \mathrm{~mL}$ boric acid (pH9.0, 0.2M) and $0.1 \mathrm{~mL}$ Tween20. Then, $0.2 \mathrm{~mL} \mathrm{NaOH}(1 \mathrm{M})$ was added and mixed until a clear solution was obtained. Finally, boric acid was added to increase the solution volume to $40 \mathrm{~mL}$. The substrate solution was stored at $20^{\circ} \mathrm{C}$ until use.

Statistical Analysis. Data were analyzed by SPSS version 16.0 (SPSS Inc., Chicago, Il) and Pearson correlation. The Duncan's multiple-range test was used to compare OAVs of the odor-active compounds from rice samples.

\section{Results and Discussion}

The relative intensities and descriptors of odor-active compounds emanating from cooked aromatic Kam sweet Gou Cengao rice, as determined by trained assessors, are summarized in Table 2. Aldehydes constituted the most abundant group among the 16 odor-active compounds detected in cooked Gou Cengao. Indeed, 9 aldehydes were found, including hexanal, furfural, heptanal, (E)-2heptenal, octanal, $(E)-2-o c t e n a l$, nonanal, decanal, and ( $E$, $E)-2$, 4-nonadienal. The remaining constituents were two aromatic compounds (benzaldehyde and 2-pentylfuran), two alcohols (1-octen-3-ol and 1-octanol), two ketones (3octen-2-one and geranylacetone) and a nitrogencontaining compound (2-acetyl-1-pyrroline). Buttery et al. [16] reported 2-acetyl-1-pyrroline and (E, E)-2, 4decadienal as key aroma components in cooked rice, compounds not found cooked Gou Cengao as shown above. This is likely due to the difference in the aromatic rice types studied. Based on odor intensity, 2-acetyl-1pyrroline, nonanal, and hexanal contributed most to the flavor of Kam sweet rice Gou Cengao, with odor intensity values of 5.0, 5.0 and 4.2, respectively.

Table 2. Odor Intensity and Description of Odor-Active Compounds in Cooked Kam sweet rice Gou Cengao

\begin{tabular}{|c|c|c|c|c|c|}
\hline No. & $\mathrm{RI}^{\mathrm{b}}$ & Odorant & Odor intensity $^{\mathrm{a}}$ & Odor description $^{c}$ & Identification $^{\mathrm{d}}$ \\
\hline 1 & 643 & hexanal & 4.2 & Green & MS, STD, RI \\
\hline 2 & 670 & furfural & nd & nd & MS, STD, RI \\
\hline 3 & 744 & heptanal & 1.8 & floral & MS, STD, RI \\
\hline 4 & 793 & 2-acetyl-1-pyrroline & 5.0 & popcorn & MS, STD, RI \\
\hline 5 & 820 & (E)-2-heptenal & nd & nd & MS, STD, RI \\
\hline 6 & 826 & benzaldehyde & nd & nd & MS, STD, RI \\
\hline 8 & 854 & 2-pentylfuran & nd & nd & MS, STD, RI \\
\hline 9 & 869 & octanal & 3.3 & green citrus & MS, STD, RI \\
\hline 10 & 904 & 3-octen-2-one & nd & nd & MS, STD, RI \\
\hline 11 & 906 & (E)-2-octenal & 2.4 & citrus & MS, STD, RI \\
\hline 12 & 920 & 1-octanol & 0.3 & nutty fatty & MS, STD, RI \\
\hline 13 & 968 & nonanal & 5.0 & green fatty & MS, STD, RI \\
\hline 14 & 1041 & decanal & 3.2 & citrus & MS, STD, RI \\
\hline 16 & 1273 & geranylacetone & nd & nd & MS, STD, RI \\
\hline
\end{tabular}

${ }^{a}$ Average intensity of compounds that were detected by all three assessors.nd=not detected. ${ }^{b}$ Retention indexs were counted using a series of n-alkanes $\mathrm{C}_{6}-\mathrm{C}_{19}{ }^{c}$ Odorants were described by assessors during GC-O. ${ }^{d} \mathrm{MS}$, by comparison of the mass spectrum with the NIST/Wiley mass spectral library; RI, by comparison of RI with those from the literature;STD, by comparison of retention time, spectrum, and odor description of an identified compound with those of an authentic compound.

To assess the contribution of individual odor active components to the overall aroma, the compounds detected by the assessors (i.e., odor intensity $>0$ ) were considered potentially contributors (Table 3 ) $[17,18]$. OAVs, obtained by dividing each component's concentration by its odor threshold in air, were used to assess the relative importance of individual aromatic compounds in cooked rice aroma (Table 3). To determine the concentrations of the odor-active components, excellent standard curves were established using authentic standards, with linear correlation coefficients $\left(\mathrm{R}^{2}\right)$ ranging from 0.9991 to 0.9999 and relative standard deviations (RSDs) from 0.29 to $3.74 \%$ (Table 1 ). As shown in Table 3, nonanal was the most potent odor-active compound in the aromatic Kam sweet rice Gou Cengao $(\mathrm{OAV}=106)$, followed by 2acetyl-1-pyrroline $(\mathrm{OAV}=101)$, hexanal $(\mathrm{OAV}=53)$, octanal $(\mathrm{OAV}=48)$, decanal $(\mathrm{OAV}=10)$, $(E, E)-2$, 4nonadienal $(\mathrm{OAV}=9.6),(E)$-2-octenal $(\mathrm{OAV}=8.8)$, heptanal $(\mathrm{OAV}=5.6)$, 1-octen-3-ol $(\mathrm{OAV}=2.2)$, and 1 octanol $(\mathrm{OAV}=0.6)$. 
Table 3. Odor Activity Values(OAVs) of Major Odor-Active Compounds in Cooked aromatic Kam sweet rice Gou Cengao

\begin{tabular}{|c|c|c|c|}
\hline $\mathrm{RI}^{\mathrm{b}}$ & odorant & $\mathrm{OAV}^{\mathrm{a}}(\mathrm{n}=3)$ & odor threshold in air(ng/L) \\
\hline 643 & hexanal & $53 \mathrm{f}$ & $1.1^{\mathrm{c}}$ \\
\hline 744 & heptanal & $5.6 \mathrm{bc}$ & $0.9^{c}$ \\
\hline 793 & 2-acetyl-1-pyrroline & $101 \mathrm{~g}$ & $0.02^{\mathrm{d}}$ \\
\hline 849 & 1-octen-3-ol & 2.2ab & $2.7^{\mathrm{c}}$ \\
\hline 869 & octanal & $48 \mathrm{e}$ & $0.4^{\mathrm{c}}$ \\
\hline 906 & (E)-2-octenal & $8.8 \mathrm{~cd}$ & $2.7^{\mathrm{c}}$ \\
\hline 920 & 1-octanol & $0.6 \mathrm{a}$ & $22^{c}$ \\
\hline 968 & nonanal & $106 \mathrm{~h}$ & $2.6^{\mathrm{c}}$ \\
\hline 1041 & decanal & 10d & $2.6^{\mathrm{c}}$ \\
\hline 1075 & (E, E)-2, 4-nonadienal & 9.6cd & $0.2^{\mathrm{c}}$ \\
\hline
\end{tabular}

${ }^{a} \mathrm{OAV}$ is obtained by dividing the concentration of an odor-active compound by its odor threshold in air and means of three replicates;means separation within rows by Duncan's multiple-range test $P<0.05 .{ }^{b}$ Retention indexs were counted using a series of n-alkanes $\mathrm{C}_{6}-\mathrm{C}_{19}{ }^{c}{ }^{c}$ Data from Yang(15). ${ }^{d}$ Data from Schieberle(16).

Aldehydes, especially nonanal $(\mathrm{OAV}=106$, relative proportion $=30.7 \%)$, constituted the most odor active contributors of the aromatic Kam sweet rice Gou Cengao. Interestingly, C9-aldehydes are significant odorants in many rice types and known products of 9-lipoxygenase and 9-hydroperoxide lyase, which are encoded separately by OsLOX3 [19] and OsHPL1 [20] gene, respectively.

Therefore, to understand the highest OAV of nonanal, we assessed the changes in nonanal concentrations (Table 4), relative gene expression of OsLOX3 and OsHPL1 (Figure 1A and Figure 1B; Table 5), lipoxygenase activity (Table 4; Figure 2) and ABA concentrations (Table 5) in grains 6, 8, 10, and 15 days after flowering of aromatic Kam sweet rice Gou Cengao. As shown in Table 4, nonanal was found at all stages of grain development in aromatic Kam sweet rice Gou Cengao. Interestingly, OsLOX3 expression was significantly higher in Gou Cengao than in Lailong at all grain filling stages (Figure 1A). However, no constant trend was found for OsHPL1 expression, which was lower in Gou Cengao compared with Lailong at 6 (0.207 vs. 1.895), 8 (1.613 vs. 2.288) and 15 days (0.012 vs. 0.112$)$ but slightly higher at 10 days (1.641 vs. 1.415) (Figure 1B.). Therefore, OsLOX3 was considered the most critical gene in aromatic Kam sweet rice Gou Cengao. To confirm these findings,

enzyme activity of lipoxygenase was assessed in the same samples (Table 4; Figure 2). In agreement with gene expression data, lipoxygenase activity was markedly increased at all time points during rice filling. Finally, using Pearson correlation analysis, we found that variation in nonanal amounts correlated with the enzyme activity of lipoxygenase, with a significant correlation of $r=0.694$ (two-tailed analysis, $p=0.012$ ). However, the variation in ABA amounts correlated with the OsLOX3 expression, with a significant negative correlation of $r=-0.945$ (twotailed analysis, $p<0.01$ ) (Table 5). Using Pearson correlation analysis, we found no significant difference in the correlation between ABA concentration and OsHPL1 expression, with correlation of $r=-0.421$ (two-tailed analysis, $p=0.173$ ) (Table 5). The result suggested that OsLOX3 was also the most critical gene in ABA regulation pathway of nonanal. The ABA showed the inhibiting effect on the biosynthesis of nonanal. To confirm the findings, the nonanal concentrations were also assessed in the rice seedlings after application of ABA. In agreement with the negative correlation between ABA and OsLOX3 expression, the nonanal from Gou Cengao seedlings treated by ABA was significantly lower than the seedlings from control (33.889 vs. 53.866) (Table 6).

Table 4. The quatification of nonanal and enzyme activity of lipoxygenase in Kam sweet rice during rice filling stage

\begin{tabular}{cccc}
\hline Time & quatification of nonanal $(\mathrm{ng} / \mathrm{g})$ & $\begin{array}{c}\text { enzyme activity of lipoxygenase }\left(\Delta \mathrm{A}_{234} \mathrm{~min}^{-1} \mathrm{~g}^{-1} \mathrm{FW}\right) \\
\text { Lailong as control }\end{array}$ \\
\hline 6day & $36.187 \pm 1.582$ & $0.188 \pm 0.026$ & $0.029 \pm 0.0004$ \\
8day & $55.508 \pm 2.119$ & $0.364 \pm 0.158$ & $0.003 \pm 0.00005$ \\
10day & $33.416 \pm 0.273$ & $0.177 \pm 0.002$ & $0.024 \pm 0.0004$ \\
15day & $39.534 \pm 2.988$ & $0.228 \pm 0.028$ & $0.009 \pm 0.0001$ \\
\hline
\end{tabular}

${ }^{a}$ Time, sampling after rice flowering.

Table 5. The quatification of ABA and the relative expression value of OsLOX3 and OsHPL1 in grains during rice filling stage

\begin{tabular}{cccccc}
\hline \multirow{2}{*}{ Time $^{a}$} & \multirow{2}{*}{ quatification of ABA (ng/g) } & \multicolumn{2}{c}{ relative expression value of OsLOX3 } & \multicolumn{2}{c}{ relative expression value of OsHPL1 } \\
& & aromatic Gou Cengao & Lailong as control & aromatic Gou Cengao & Lailong as control \\
\hline 6day & $37.427 \pm 3.223$ & $41.757 \pm 0.629$ & $7.890 \pm 0.735$ & $0.207 \pm 0.008$ & $1.895 \pm 0.168$ \\
8day & $322.873 \pm 17.874$ & $27.091 \pm 0.275$ & $0.335 \pm 0.058$ & $1.613 \pm 0.015$ & $2.288 \pm 0.354$ \\
10day & $72.625 \pm 3.431$ & $46.953 \pm 6.911$ & $5.549 \pm 0.181$ & $1.641 \pm 0.014$ & $1.415 \pm 0.232$ \\
15day & $138.816 \pm 10.458$ & $6.377 \pm 0.825$ & $0.374 \pm 0.072$ & $0.012 \pm 0.001$ & $0.112 \pm 0.009$ \\
\hline
\end{tabular}

${ }^{a}$ Time, sampling after rice flowering.

Table 6. The quatification of nonanal in air from the leaves of Gou Cengao seedlings during ABA (110mg/L, 48h) treatment

\begin{tabular}{ccccc}
\hline RI $^{\mathrm{a}}$ & Odorant & ABA-treated seedlings $(\mathrm{ng} / \mathrm{L})$ & Control (ng/L) & Odor description $^{\mathrm{b}}$ \\
\hline 968 & nonanal & $33.889 \pm 1.651$ & $53.866 \pm 0.825$ & Identification $^{\mathrm{c}}$ \\
\hline${ }^{a}$ Retention indexs were counted using a series of n-alkanes $\mathrm{C}_{6}-\mathrm{C}_{19} \cdot{ }^{b}$ Odorants were described by assessors during GC-O. ${ }^{c}$ MS, by comparison of the
\end{tabular}

${ }^{a}$ Retention indexs were counted using a series of n-alkanes $\mathrm{C}_{6}-\mathrm{C}_{19} \cdot{ }^{b}$ Odorants were described by assessors during GC-O. ${ }^{c} \mathrm{MS}$, by comparison of the mass spectrum with the NIST/Wiley mass spectral library;RI, by comparison of RI with those from the literature;STD, by comparison of retention time, spectrum, and odor description of an identified compound with those of an authentic compound. 
A

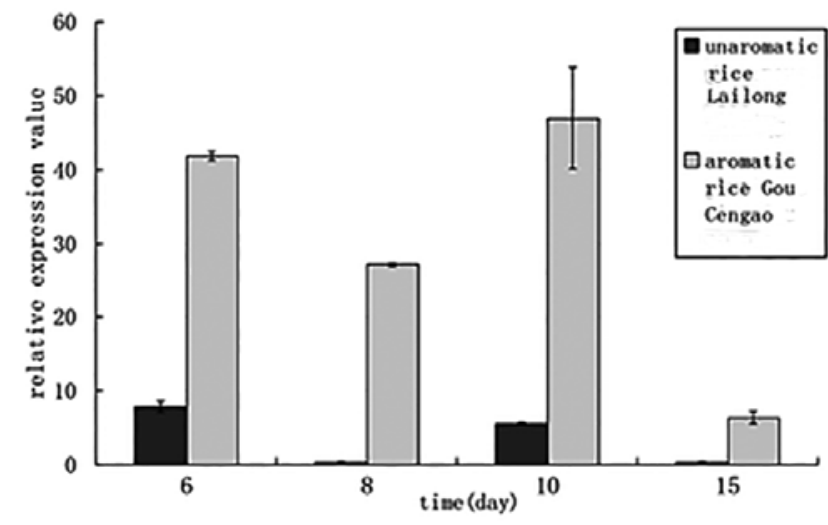

B

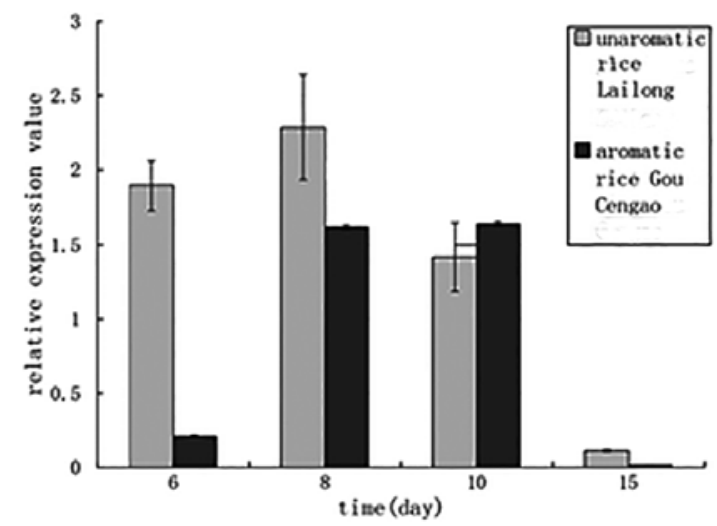

Figure 1. Relative gene expression of OsLOX3 and OsHPL1 in aromatic Kam sweet rice Gou Cengao and non-aromatic Kam sweet rice Lailong during rice filling stages. A, OsLOX3 gene expression; B, OsHPL1 gene expression

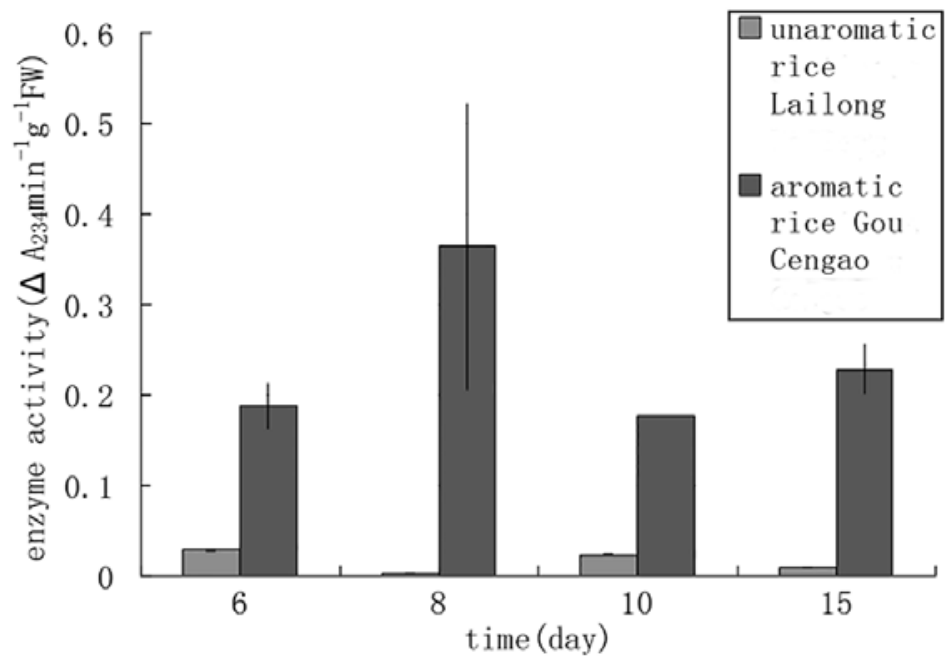

Figure 2. The comparison of lipoxygenase enzyme activity between the aromatic Kam sweet rice Gou Cengao and non-aromatic Kam sweet rice Lailong during rice filling stage

\section{Conclusions}

In this study, the characteristic aroma profile of aromatic Kam sweet rice Gou Cengao was determined by a combination of GC-O and GC-MS. Nonanal and 2acetyl-1-pyrroline were considered the most potent flavor compounds, with nonanal displaying the highest OAV. Moreover our data showed that OsLOX3 gene was expressed in relatively high levels in aromatic rice and this translated into high lipogenase activity. A positive correlation was also found between nonanal concentrations and lipoxygenase activity, suggesting that the high nonanal OAV observed results from elevated lipoxygenase activity in Gou Cengao rice. The inhibiting effect of ABA on nonanal concentrations was proved by the decrease in nonanal of rice seedlings after application of ABA. A negative correlation was also showed by the Pearson correlation between the endogenous ABA and OsLOX3 gene expression. Interestingly, the novelty of the study lied in the finding contrasted with the past finding that ABA elevated the aroma content of aromatic rice in ABAtreated leaves of rice [5]. The reason caused the opposite result might be the difference between the effects of $\mathrm{ABA}$ on the metabolic pathways of nonanal and 2-acetyl-1pyrroline. The present results suggested that rice of different origins might have also differences in hormonal regulation.

\section{Acknowledgments}

The authors are grateful to the National Transgenic Major Project of China (2011ZX08010-003) and the Province Science Project in Guizhou (Z [2012] 4008) for their financial support.

\section{References}

[1] Choudhury B, Khan ML, Dayanandan S (2013) Genetic structure and diversity of indigenous rice (Oryza sativa) varieties in the Eastern Himalayan region of Northeast India. SpringerPlus 2: 1-10.

[2] Stone R (2008) Chinese Province Crafts Pioneering Law to Thwart Biopiracy. Science 320: 732-733.

[3] Olsen KM, Purugganan MD (2002) Molecular evidence on the origin and evolution of glutinous rice. Genetics 162: 941-950.

[4] Piebiep G, Sugunya W, Xiangru T (2011) Decrease in rice aroma after application of growth regulators. Agron 31: 349-359.

[5] Roychoudhury A, Basu S, Sengupta DN (2009) Effects of exogenous abscisic acid on some physiological responses in a popular aromatic indica rice compared with those from two traditional non-aromatic indica rice cultivars. Acta Physiologiae Plantarum 31: 915-926. 
[6] Yoshihashi T, Nguyen TTH, Kabaki N (2004) Area dependency of 2-acetyl-1-pyrroline content in an aromatic rice variety, Khao Dawk Mali 105. Japan Agricultural Research Quarterly 38: 105110.

[7] Gay F, Maraval I, Roques S, Gunata Z, Boulanger R, Audebert A, Mestres C (2010) Effect of salinity on yield and 2-acetyl-1pyrroline content in the grains of three fragrant rice cultivars (Oryza sativa L.) in Camargue (France). Field crops research 117: 154-160.

[8] Fitzgerald TL, Waters DLE, Brooks LO, Henry RJ (2010) Fragrance in rice ( Oryza sativa) is associated with reduced yield under salt treatment. Environmental and Experimental Botany 68: 292-300.

[9] Poonlaphdecha J, Maraval I, Roques S, Audebert A, Boulanger R, Bry X, Gunata Z (2012) Effect of Timing and Duration of Sal Treatment during Growth of a Fragrant Rice Variety on Yield and 2-Acetyl-1-pyrroline, Proline, and GABA Levels. Journal of agricultural and food chemistry 60: 3824-3830.

[10] Huang TC, Teng CS, Chang JL, Chuang HS, Ho CT, Wu ML (2008) Biosynthetic mechanism of 2-acetyl-1-pyrroline and its relationship with Delta1-pyrroline-5-carboxylic acid and methylglyoxal in aromatic rice (Oryza sativa L.) callus. J Agric Food Chem 56:7399-7404.

[11] Ratchatachaiyos K, Theerakulkait C (2009) Isolation and some characteristics of Lipoxygenase from aromatic brown rice (Oryza sativa L.) cv. Khao Dawk Mali 105. Journal of immunoassay \& immunochemistry 30: 106-117.
[12] Itani T, Tamaki M, Hayata Y, Fushimi T, Hashizume K (2004) Variation of 2-acetyl-1-pyrroline concentration in aromatic rice grains collected in the same region in Japan and factors affecting its concentration. Plant production science 7: 178-183.

[13] Yang DS, Lee KS, Jeong OY, Kim KJ, Kays SJ (2008) Characterization of volatile aroma compounds in cooked black rice. J Agric Food Chem 56: 235-240.

[14] Suzuki Y, Matsukura U (1997) Lipoxygenase activity in maturing and germinating rice seeds with and without lipoxygenase- 3 in mature seeds. Plant science 125: 119-126.

[15] Engeseth N, Klein B, Warner K (1987) Lipoxygenase lsoenzymes in Soybeans: Effects on Crude Oil Quality. Journal of Food Science 52: 1015-1019.

[16] Buttery RG, Turnbaugh JG, Ling LC (1988) Contribution of volatiles to rice aroma. Journal of agricultural and food chemistry 36: 1006-1009.

[17] Yang DS, Shewfelt RL, Lee K-S, Kays SJ (2008) Comparison of odor-active compounds from six distinctly different rice flavor types. Journal of agricultural and food chemistry 56:2780-2787

[18] Schieberle P (1991) Primary odorants in popcorn. Journal of agricultural and food chemistry 39: 1141-1144

[19] Marla SS, Singh VK (2012) LOX genes in blast fungus (Magnaporthe grisea) resistance in rice. Functional \& integrative genomics 12:265-275.

[20] Chehab EW, Raman G, Walley JW, Perea JV, Banu G, Theg S, Dehesh K (2006) Rice HYDROPEROXIDE LYASES with unique expression patterns generate distinct aldehyde signatures in Arabidopsis. Plant physiology 141: 121-134. 